\title{
Multi space RB preconditioners for large-scale parametrized PDEs
}

\author{
Niccolò Dal Santo*
}

* École Polytechnique Fédérale de Lausanne, CMCS, Institute of Mathematics, Switzerland niccolo.dalsanto@epfl.ch

\section{The main ideas}

Goal: speed up the iterative solution of parametrized finite element linear systems with a preconditioner which exploits the reduced basis method.

Preconditioning
techniques

HPC OPTIMALITY
SCALABILITY

Multi space reduced basis preconditioners

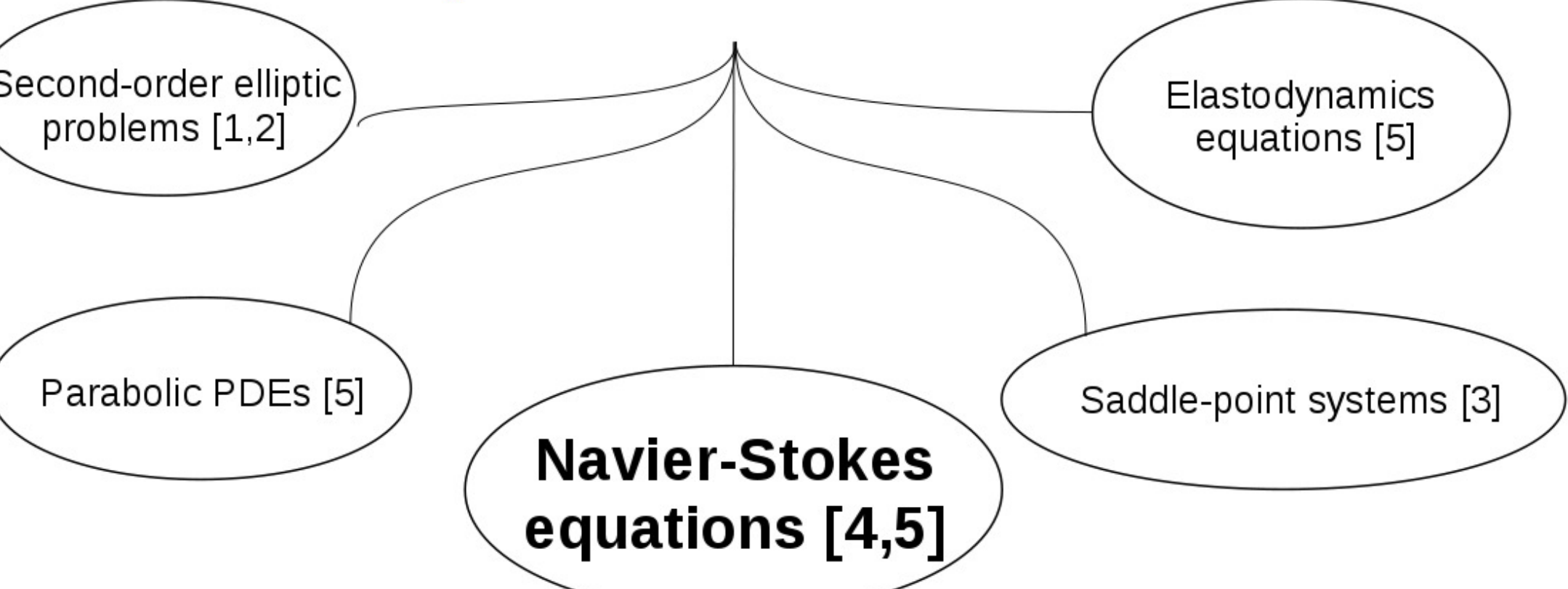

- Efficient solution of the linear systems arising from the finite element (FE) discretization of parametrized PDEs.

- Focus on the parametrized Navier-Stokes (NS) equations.

- Combination of a fine grid preconditioner with a reduced basis (RB) coarse component to exploit the parameter dependence. - Introduction of a new Multi space RB method for NS equations: - Efficient treatment of the linearized term of the NS equations. - Approximated well-posedness of the underlying RB coarse component through a velocity-enrichment strategy.

\section{The parametrized unsteady Navier-Stokes problem}

Consider the NS equations in a $\boldsymbol{\mu}$-dependent domain $\Omega(\boldsymbol{\mu})$

$\begin{cases}\frac{\partial \vec{u}(\boldsymbol{\mu})}{\partial t}+\vec{u}(\boldsymbol{\mu}) \cdot \nabla \vec{u}(\boldsymbol{\mu})-\nabla \cdot \boldsymbol{\sigma}(\boldsymbol{\mu})=\overrightarrow{0} & \text { in } \Omega(\boldsymbol{\mu}) \times(0, T) \\ \nabla \cdot \vec{u}(\boldsymbol{\mu})=0 & \text { in } \Omega(\boldsymbol{\mu}) \times(0, T) \\ + \text { b.c. } & \\ + \text { i.c. } & \end{cases}$

$\boldsymbol{\sigma}(\boldsymbol{\mu})=\boldsymbol{\sigma}(\vec{u}(\boldsymbol{\mu}), p(\boldsymbol{\mu}))=-p(\boldsymbol{\mu}) \mathbf{I}+\nu\left(\nabla \vec{u}(\boldsymbol{\mu})+\nabla \vec{u}(\boldsymbol{\mu})^{T}\right)$

The FE method and a semi-implicit time advance scheme yield for any $\boldsymbol{\mu} \in \mathcal{D}$ and for any $n=0, \ldots, N_{t}-1$, the linear system

$\underbrace{\left[\begin{array}{cc}\frac{\alpha_{1}}{\Delta t} \mathbf{M}^{u}(\boldsymbol{\mu})+\mathbf{D}(\boldsymbol{\mu})+\mathbf{C}\left(\mathrm{u}^{n, *}(\boldsymbol{\mu}) ; \boldsymbol{\mu}\right) & \mathbf{B}^{T}(\boldsymbol{\mu}) \\ \mathbf{B}(\boldsymbol{\mu}) & \mathbf{0}\end{array}\right]}_{\mathbf{N}\left(\mathrm{u}^{n, *}(\mu) ; \boldsymbol{\mu}\right) \in \mathbb{R}^{N_{h} \times N_{h}}} \underbrace{\left[\begin{array}{l}\mathbf{u}^{n+1}(\boldsymbol{\mu}) \\ \mathbf{p}^{n+1}(\boldsymbol{\mu})\end{array}\right]}_{\mathbf{z}^{n+1}(\boldsymbol{\mu})}=\mathbf{g}^{n+1}$

Currently employed techniques to deal with the NS system are:

- Preconditioned Krylov methods: domain decomposition, multilevel, block preconditioners (PCD, LSC, SIMPLE).

- RB methods: exploits the parameter dependence but its efficiency may deteriorate when targeting accurate approximations (due to affine approximations and RB space accuracy).

\section{How to exploit the RB method for preconditioning?}

The RB method in a nutshell: approximate $\mathbf{u}^{n}(\boldsymbol{\mu}), \mathbf{p}^{n}(\boldsymbol{\mu})$ in two $R B$ spaces $\mathbf{V}_{N_{u}}$ and $\mathbf{V}_{N_{p}}$

$$
\mathbf{u}^{n}(\boldsymbol{\mu}) \approx \mathbf{V}_{N^{\prime}} \mathbf{u}_{N}^{n}(\boldsymbol{\mu}), \quad \mathbf{p}^{n}(\boldsymbol{\mu}) \approx \mathbf{V}_{N_{N}} \mathbf{p}_{N}^{n}(\boldsymbol{\mu})
$$

- offline: build $\mathbf{V}_{N_{u}}, \mathbf{V}_{N_{p}}$ through POD by FE snapshots and up to a prescribed tolerance $\varepsilon_{\mathrm{POD}}$ (e.g. with a double POD-approach), then $\mathbf{V}=\operatorname{diag}\left(\mathbf{V}_{N_{N}}, \mathbf{V}_{N_{p}}\right)$.

! Warning: $\mathbf{V}_{N_{u}}$ must be enriched with a supremizer basis to obtain an (approximately) inf-sup stable RB formulation.

- online: given a new $\boldsymbol{\mu}$, for any $n$ solve a small RB problem:

$$
\underbrace{\mathbf{V}^{T} \mathbf{N}\left(\mathbf{u}^{n, *}(\boldsymbol{\mu}) ; \boldsymbol{\mu}\right) \mathbf{V}}_{\mathbf{N}_{N}\left(\mathbf{V}_{N_{u}} \mathbf{u}_{N}^{n * *}(\boldsymbol{\mu}) ; \boldsymbol{\mu}\right)} \underbrace{\left[\begin{array}{l}
\mathbf{u}_{N}^{n+1}(\boldsymbol{\mu}) \\
\mathbf{p}_{N}^{n+1}(\boldsymbol{\mu})
\end{array}\right]}_{\mathbf{z}_{N}^{n+1}(\boldsymbol{\mu})}=\underbrace{\mathbf{V}^{T} \mathbf{g}^{n+1}(\boldsymbol{\mu})}_{\mathbf{g}_{N}^{n+1}(\boldsymbol{\mu})},
$$

- See $[4,5]$ for the stability and the efficient construction of (1).

A RB low-rank solver $\mathbf{Q}_{N}(\mu)$ s.t.

$$
\begin{aligned}
\mathbf{z}^{n+1}(\boldsymbol{\mu}) & \approx \mathbf{V z}_{N}^{n+1}(\boldsymbol{\mu})=\mathbf{Q}_{N}(\boldsymbol{\mu}) \mathbf{g}^{n+1}(\boldsymbol{\mu}) \\
& =\mathbf{V N}_{N}^{-1}\left(\mathbf{V}_{N_{u}} \mathbf{u}_{N}^{n * *}(\boldsymbol{\mu}) ; \boldsymbol{\mu}\right) \mathbf{V}^{\top} \mathbf{g}^{n+1}(\boldsymbol{\mu}),
\end{aligned}
$$

we use $\mathbf{Q}_{N}(\boldsymbol{\mu})$ as coarse component in a two level preconditioner $\mathbf{Q}(\boldsymbol{\mu})$ with $\mathbf{P}(\boldsymbol{\mu}) \in \mathbb{R}^{N_{h} \times N_{h}}$ as fine component, s.t.

$\mathbf{Q}^{-1}(\boldsymbol{\mu})=\mathbf{P}^{-1}(\boldsymbol{\mu})+\mathbf{Q}_{N}(\boldsymbol{\mu})\left(\mathbf{I}_{N_{h}}-\mathbf{N}\left(\mathbf{u}^{n, *}(\boldsymbol{\mu}) ; \boldsymbol{\mu}\right) \mathbf{P}^{-1}(\boldsymbol{\mu})\right)$
4. Multi space reduced basis preconditioners (MSRB) [1]

! Warning: At iteration $k$ of GMRES, $\mathbf{Q}^{-1}(\boldsymbol{\mu})$ is applied to the k-th Krylov basis $\mathbf{v}_{k}^{n}=\mathbf{v}_{k}^{n}(\boldsymbol{\mu})$, that is

$\mathbf{Q}^{-1}(\boldsymbol{\mu}) \mathbf{v}_{k}^{n}=\mathbf{P}^{-1}(\boldsymbol{\mu})+\mathbf{Q}_{N}(\boldsymbol{\mu}) \underbrace{}_{\mathbf{I}_{h}}-\mathbf{N}\left(\mathbf{u}^{n, *}(\boldsymbol{\mu}) ; \boldsymbol{\mu}\right) \mathbf{P}^{-1}(\boldsymbol{\mu})) \mathbf{v}_{k}^{n}$

$\Rightarrow$ the RB low-rank solver must be trained to accurately solve

$\mathbf{N}\left(\mathbf{u}^{n, *}(\boldsymbol{\mu}) ; \boldsymbol{\mu}\right) \mathbf{y}_{k}^{n}(\boldsymbol{\mu})=\mathbf{v}_{k+\frac{1}{2}}^{n}(\boldsymbol{\mu})$

a NS system with solution $\mathbf{y}_{k}^{n}(\boldsymbol{\mu})=\left[\mathbf{y}_{u, k}^{n}(\boldsymbol{\mu}), \mathbf{y}_{p, k}^{n}(\boldsymbol{\mu})\right]^{T}$

The key idea: compute from (2) a set of snapshots $\left\{\mathbf{y}_{k}^{n}\left(\boldsymbol{\mu}_{i}\right)\right\}_{i=1, n=1}^{n_{s}, N_{t}}$ and build with POD a sequence of $k$-dependent RB spaces $k=1,2, \ldots$ to approximate the velocity $\mathbf{y}_{u, k}^{n}(\boldsymbol{\mu})$ and pressure $\mathbf{y}_{p, k}^{n}(\boldsymbol{\mu})$

$$
\begin{gathered}
\mathbf{v}_{u k}=\operatorname{POD}\left(\left[\mathbf{y}_{u, k}^{n}\left(\boldsymbol{\mu}_{i}\right)\right]_{i=1, n=1}^{n_{s}, N_{t}}, \varepsilon_{\mathrm{POD}}^{k}\right), \\
\mathbf{v}_{p k}=\operatorname{POD}\left(\left[\mathbf{y}_{p, k}^{n}\left(\boldsymbol{\mu}_{i}\right)\right]_{i=1, n=1}^{n_{s}, N_{t}}, \varepsilon_{\mathrm{POD}}^{k}\right), \\
\mathbf{v}_{k}=\operatorname{diag}\left(\mathbf{v}_{u k}, \mathbf{v}_{p k}\right) .
\end{gathered}
$$

$\ln \mathbf{V}_{k}$ an accurate RB approximation to $\mathbf{y}_{k}(\boldsymbol{\mu})$ is found Multi space reduced basis (MSRB) preconditioner Key fact: each RB space is constructed up to a tolerance $\varepsilon_{\mathrm{POD}}^{k}$ and the error decreases of a factor $\varepsilon_{\mathrm{POD}}^{k}$ at iteration $k$.

To each $\mathbf{V}_{k}$ we associate

$\mathbf{N}_{N_{k}}\left(\mathbf{u}^{n, *}(\boldsymbol{\mu}) ; \boldsymbol{\mu}\right)=\mathbf{V}_{k}^{T} \mathbf{N}\left(\mathbf{u}^{n, *}(\boldsymbol{\mu}) ; \boldsymbol{\mu}\right) \mathbf{V}_{k}$

$$
\begin{gathered}
=\left[\frac{\alpha_{1}}{\Delta t} \mathbf{M}_{N_{k}}^{u}(\boldsymbol{\mu})+\mathbf{D}_{N_{k}}(\boldsymbol{\mu})+\mathbf{C}_{N_{k}}\left(\mathbf{u}^{n, *}(\boldsymbol{\mu}) ; \boldsymbol{\mu}\right)\right. \\
\mathbf{B}_{N_{k}}^{T}(\boldsymbol{\mu}) \\
\mathbf{Q}_{N_{k}}(\boldsymbol{\mu})=\mathbf{V}_{k} \mathbf{N}_{N_{k}}^{-1}\left(\mathbf{u}^{n, *}(\boldsymbol{\mu}) ; \boldsymbol{\mu}\right) \mathbf{V}_{k}^{T},
\end{gathered}
$$

The multiplicative combination of $\mathbf{P}^{-1}(\boldsymbol{\mu})$ and $\mathbf{Q}_{N_{k}}(\boldsymbol{\mu})$ leads to the $k$-dependent operator

$\mathbf{Q}_{\mathrm{MSRB}, k}(\boldsymbol{\mu})=\mathbf{P}^{-1}(\boldsymbol{\mu})+\mathbf{Q}_{N_{k}}(\boldsymbol{\mu})\left(\mathbf{I}_{N_{h}}-\mathbf{N}\left(\mathbf{u}^{n, *}(\boldsymbol{\mu}) ; \boldsymbol{\mu}\right) \mathbf{P}^{-1}(\boldsymbol{\mu})\right)$ Key fact: $\mathbf{Q}_{\mathrm{MSRB}, k}(\boldsymbol{\mu})$ is iteration dependent $\Rightarrow$ flexible GMRES.

Stability of the RB coarse operators: (3) does not guarantee $\overline{\mathbf{N}}_{N_{k}}\left(\mathbf{u}^{n, *}(\boldsymbol{\mu}) ; \boldsymbol{\mu}\right)$ to be nonsingular $\Rightarrow$ velocity enrichment strategy: to each $\mathbf{y}_{p, k}^{n}\left(\boldsymbol{\mu}_{i}\right)$ we associate the supremizer $\mathbf{y}_{t, k}^{n}\left(\boldsymbol{\mu}_{i}\right)$ s.t.

$$
\mathbf{X}_{h u}\left(\boldsymbol{\mu}_{i}\right) \mathbf{y}_{t, k}^{n}\left(\boldsymbol{\mu}_{i}\right)=\mathbf{B}^{T}\left(\boldsymbol{\mu}_{i}\right) \mathbf{y}_{p, k}^{n}\left(\boldsymbol{\mu}_{i}\right),
$$

with $\mathbf{X}_{h u}$ the $H_{0}^{1}$ scalar product matrix; then, we set

$$
\mathbf{v}_{s k}=\operatorname{POD}\left(\left[\mathbf{y}_{t, k}^{n}\left(\boldsymbol{\mu}_{i}\right)\right]_{i=1, n=1}^{n_{s}, N_{t}}, \varepsilon_{\mathrm{POD}}^{k}\right) .
$$

and use it to augment the velocity space

$$
\mathbf{V}_{u k} \leftarrow \text { Gram-Schmidt }\left(\left[\mathbf{V}_{u k}, \mathbf{V}_{s k}\right]\right)
$$

Efficient assembly of the RB coarse operators: Matrix Discrete Empirical Interpolation Method (MDEIM) is used to efficiently assemble $\mathbf{N}_{N_{k}}\left(\mathbf{u}^{n, *}(\boldsymbol{\mu}) ; \boldsymbol{\mu}\right)$ :

$$
\mathbf{N}_{N_{k}}\left(\mathbf{u}^{n, *}(\boldsymbol{\mu}) ; \boldsymbol{\mu}\right) \approx \widetilde{\mathbf{N}}_{N_{k}}\left(\mathbf{u}^{n, *}(\boldsymbol{\mu}) ; \boldsymbol{\mu}\right)
$$

$$
=\left[\begin{array}{cc}
\frac{\alpha_{1}}{\Delta t} \widetilde{\mathbf{M}}_{N_{k}}^{\vec{u}}(\boldsymbol{\mu})+\widetilde{\mathbf{D}}_{N_{k}}(\boldsymbol{\mu})+\widetilde{\mathbf{C}}_{N_{k}}\left(\mathbf{u}^{n, *}(\boldsymbol{\mu}) ; \boldsymbol{\mu}\right) & \widetilde{\mathbf{B}}_{N_{k}}^{T}(\boldsymbol{\mu}) \\
\widetilde{\mathbf{B}}_{N_{k}}(\boldsymbol{\mu}) & 0
\end{array}\right],
$$

by replacing the blocks with the MDEIM affinely approximated ones: - MDEIM used in a standard way on the linear terms $\mathbf{M}_{N_{k}}^{u}(\boldsymbol{\mu})$ $\mathbf{D}_{N_{k}}(\boldsymbol{\mu})$, and $\mathbf{B}_{N_{k}}(\boldsymbol{\mu})$;

- double-MDEIM strategy to affinely approximate $\mathbf{C}_{N_{k}}\left(\mathbf{u}^{n, *}(\boldsymbol{\mu}) ; \boldsymbol{\mu}\right)$ : 1. for any $\boldsymbol{\mu}_{i}$, build a MDEIM in time basis $\mathbf{C}\left(\mathbf{u}^{n, *}\left(\boldsymbol{\mu}_{i}\right), \boldsymbol{\mu}_{i}\right)$; 2. gather the MDEIM in time bases to construct a final MDEIM in parameter and affinely approximate $\mathbf{C}_{N_{k}}\left(\mathbf{u}^{n, *}(\boldsymbol{\mu}) ; \boldsymbol{\mu}\right)$

- approximated RB coarse component $\mathbf{Q}_{N_{k}}(\boldsymbol{\mu}) \approx \widetilde{\mathbf{Q}}_{N_{k}}(\boldsymbol{\mu})$, leading to an affinely approximated MSRB preconditioner

$\widetilde{\mathbf{Q}}_{\mathrm{MSRB}, k}(\boldsymbol{\mu})=\mathbf{P}^{-1}(\boldsymbol{\mu})+\widetilde{\mathbf{Q}}_{N_{k}}(\boldsymbol{\mu})\left(\mathbf{I}_{N_{h}}-\mathbf{N}\left(\mathbf{u}^{n, *}(\boldsymbol{\mu}) ; \boldsymbol{\mu}\right) \mathbf{P}^{-1}(\boldsymbol{\mu})\right.$

Key fact: a coarser affine approximation than the one used for the standard RB method can be used without affecting the local accuracy and hence the overall convergence.

Nonsingularity of the preconditioner: for any $\boldsymbol{\mu} \in \mathcal{D}$, assume that $\mathbf{P}(\boldsymbol{\mu}) \in \mathbb{R}^{N_{h} \times N_{h}}$ is a nonsingular matrix and the matrices $\mathbf{V}_{k}^{T} \mathbf{P}(\boldsymbol{\mu}) \mathbf{V}_{k}, \widetilde{\mathbf{N}}_{N_{k}}\left(\mathbf{u}^{n, *}(\boldsymbol{\mu}) ; \boldsymbol{\mu}\right) \in \mathbb{R}^{N_{k} \times N_{k}}$ are invertible. Then the matrix $\mathbf{Q}_{\mathrm{MSRB}, k}(\boldsymbol{\mu})$ is nonsingular.

Dealing with the time dependence: algorithms are developed in a time slab framework, where $[0, T]$ is divided in time slabs and a MSRB preconditioner for each time slab is constructed.

\section{Numerical results}

- Results produced with rb-LifeV, the module of LifeV (www.lifev.org) forRB methods for parametrized problems.

\section{LIFEV}

- Computational resources have been provided by the Swiss Nationa Supercomputing Center (www.cscs.ch), project ID s796. Blood flow in parametrized carotid bifurcations: Consider the NS system with final time $T=0.64 \mathrm{~s}$, viscosity $\nu=0.035 \mathrm{~cm}^{2} \mathrm{~s}^{-1}$ and $\boldsymbol{\mu}=\left(\mu_{1}, \mu_{2}\right) \in[0.2,0.4] \times[0.85,1]$ :

- $\mu_{1}$ narrows the bifurcation section by a displacement which extends harmonically a parametrized load $h(\boldsymbol{\mu})=h\left(\mu_{1}\right)$ applied on the region $A$.

- $\mu_{2}$ scales the inlet flowrate $Q_{C C A}(t ; \boldsymbol{\mu})=\mu_{2} Q_{C C A}^{0}(t)$

- A $5 \times 4$ a tensorial grid with is used train the RB coarse operators.

- SIMPLE is used as fine operator $\mathbf{P}(\boldsymbol{\mu})$ in MSRB preconditioner

- 5 RB coarse operators with $N_{k}=220$ basis function for velocity,

pressure and supremizer (dimension $3 N_{k}=660$ in total).

- $\mathcal{P}_{2}-\mathcal{P}_{1}$ FE for $\vec{u}(\boldsymbol{\mu})$ and $p(\boldsymbol{\mu})\left(N_{h}=259^{\prime} 930\right)$.

- Simulation on 32 cores on Cray XC40 computing nodes.

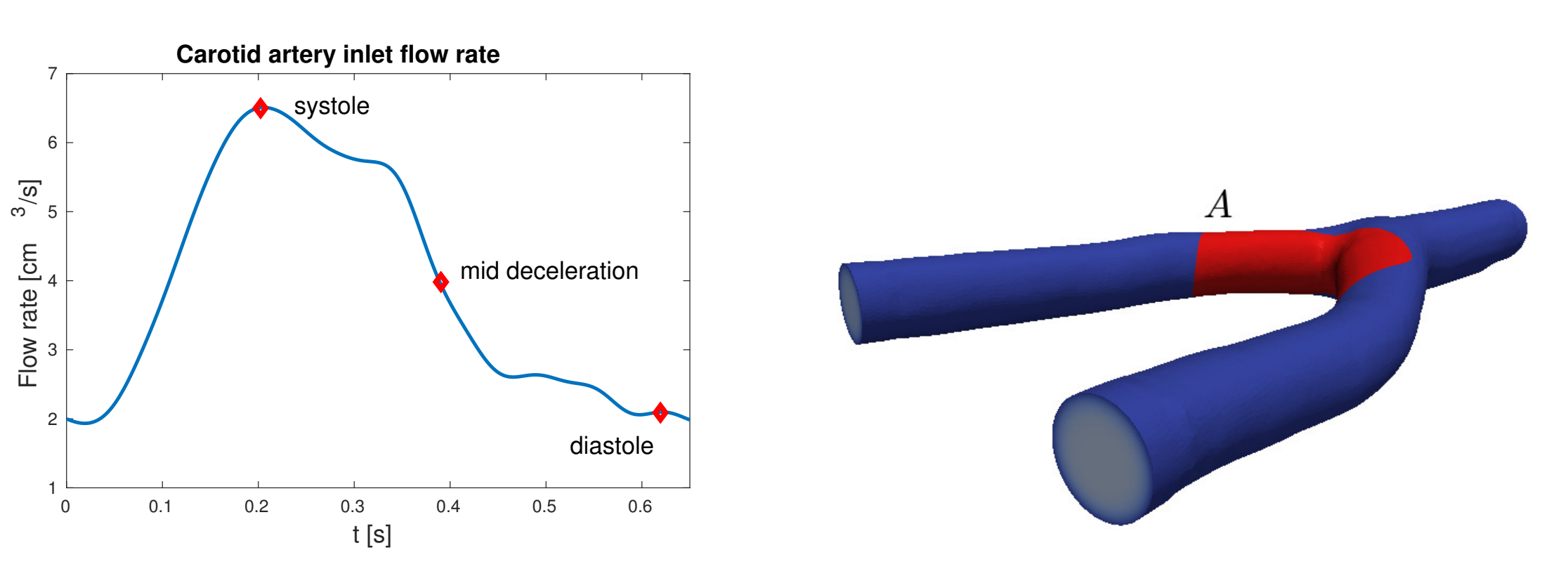

Figure 3: Reference flow rate $Q_{C C A}^{0}(t)$ and application area $A$ of $h(\boldsymbol{\mu})$.

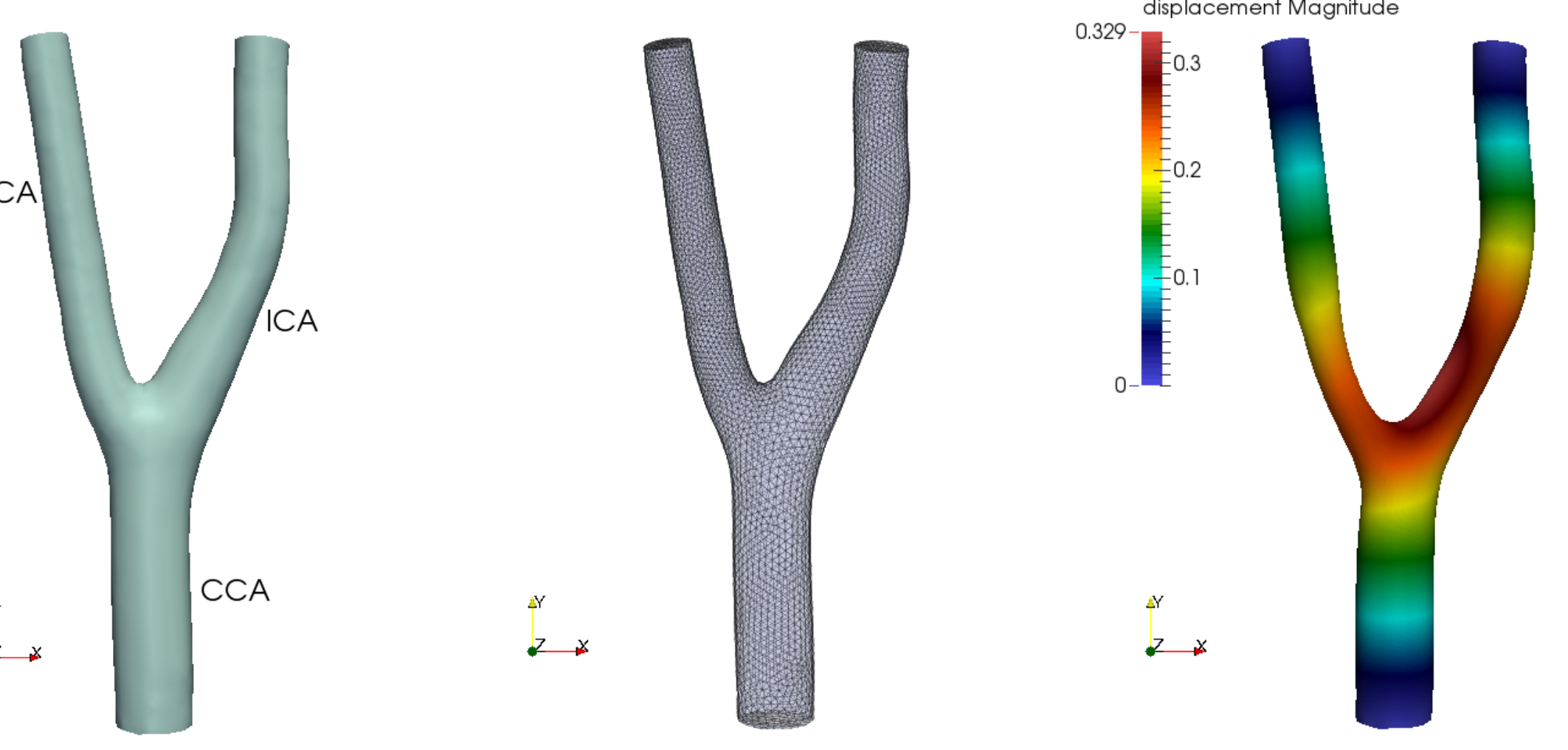

Figure 4: Carotid bifurcation, reference mesh and displacement example.

- Competitive computational time per timestep with MSRB preconditioner $\left(t_{\mathrm{MSRB}}^{\mathrm{onl}}\right)$ compared to SIMPLE preconditioner ( $\left.t_{\mathrm{SIMPLE}}\right)$. - The average iteration count per timestep significantly lower with MSRB preconditioner ( $\left.I t_{\text {MSRB }}^{\text {On }}\right)$ than using only SIMPLE preconditioner $\left(/ t_{\text {SIMPLE }}\right)$.

\begin{tabular}{|c|c|c|c|c|c|}
\hline$t_{\text {MSRB }}^{\text {ond }}$ & $I t_{\text {MSRB }}^{\text {on }}$ & $t_{\text {SIMPLE }}$ & $t_{\text {SIMPLE }}$ & $t_{\text {off }}$ & Speedup \\
\hline $\mathbf{6 . 3 3}$ & $\mathbf{3}$ & 55.06 & 91 & 98074.1 & 8.68 \\
\hline
\end{tabular}

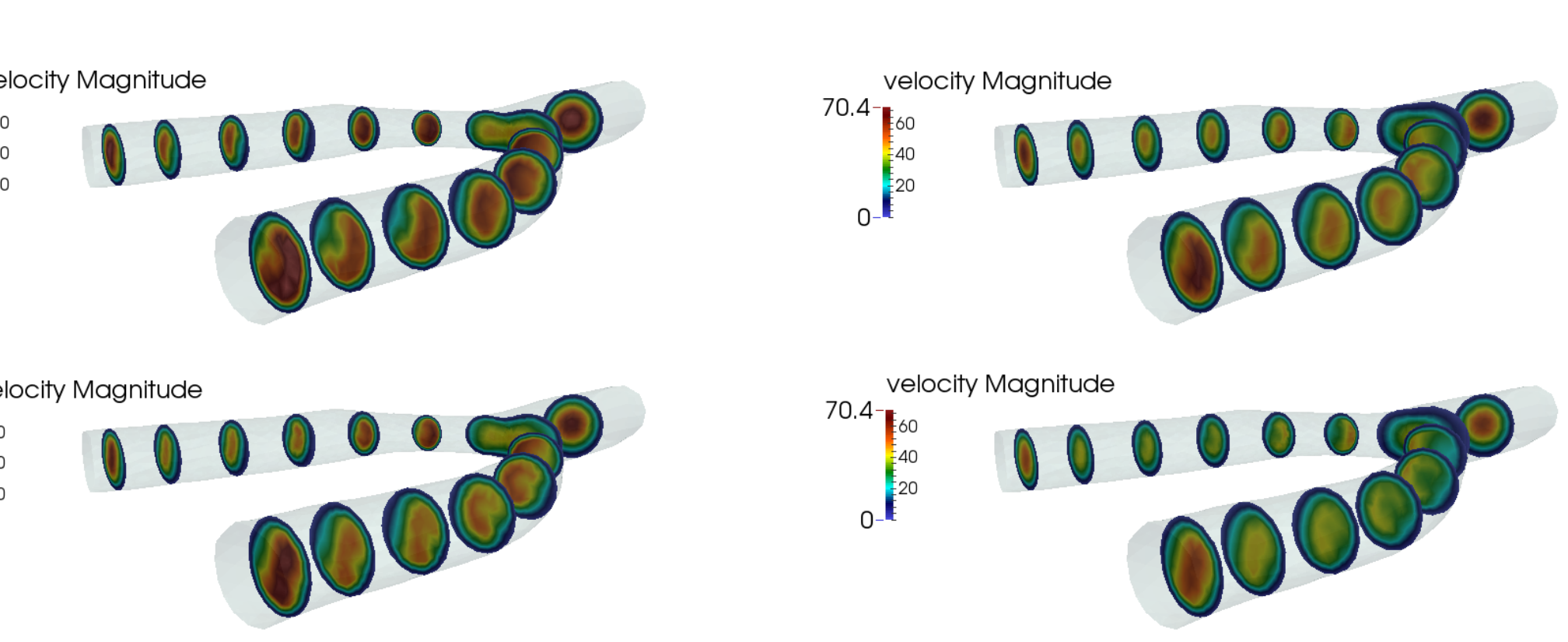

Figure 5: Velocity magnitude for $\boldsymbol{\mu}=(0.375,0.975)$ (left) $\boldsymbol{\mu}=(0.225,0.875)$ (right) at $t=0.2$ (top) and $t=0.4$ (bottom)

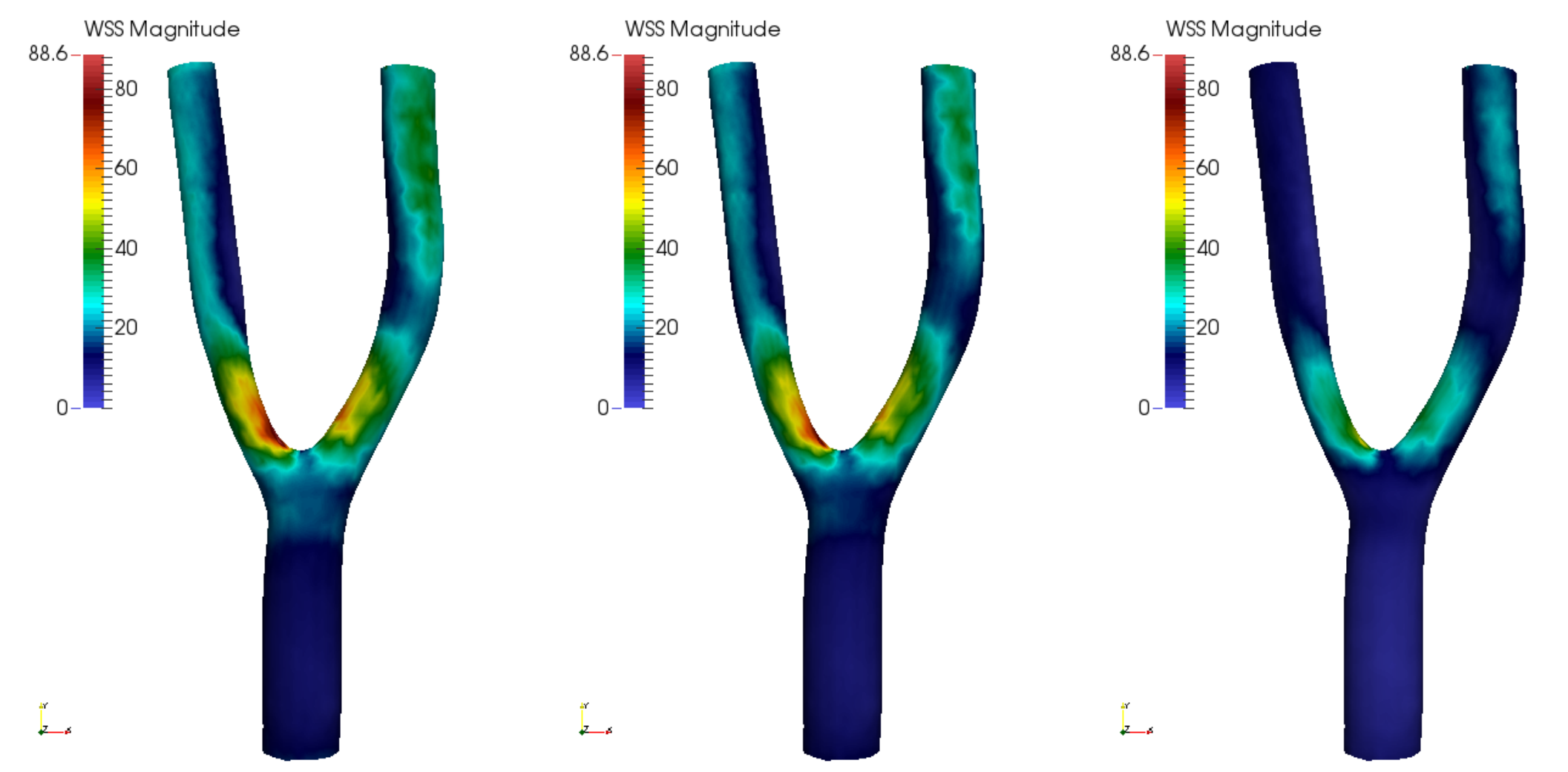

Figure 6: Wall shear stress at times $t=0.2,0.4,0.6$ for $\boldsymbol{\mu}=(0.375,0.975)$. References

1] N. Dal Santo, S. Deparis, A. Manzoni, and A. Quarteroni. Multi space reduced basis preconditioners for large-scale par
Computing, 40(2):A954-A983, 2018.

N. Dal Santo, S. Deparis and A. Manzoni A numerical investigation of multi space reduced basis preconditioners for parametrized elliptic advection-diffusion equations. reduced basis preconditioners for parametrized elliptic advection-diffusion equa
Communication in Applied and and Industrial Mathematics, 8(1):282-297. N. Dal Santo, S. Deparis, A. Manzoni, and A. Quarteroni. Multi space reduced
basis preconditioners for parametrized Stokes equations. Mathicse report 03.2018. N. Dal Santo and A. Manzoni. Hyper-reduced order models for parametrized unsteady Navier-Stokes equations on domains with variable shape. In preparation. N. Dal Santo. Multi space reduced basis preconditioners parametrized PDEs. PhD 